\title{
ON THE THEORY OF COSMIC-RAY SHOWERS I THE FURRY MODEL AND THE FLUCTUATION PROBLEM
}

\author{
by A. NORDSIECK, W. E. LAMB JR. and G. E. UHLENBECK
}

Department of Physics, Columbia University and University of Michigan.

\section{Summary}

The main problems regarding the shower formation by fast electrons are revicwed on the basis of a simplified model first proposed by $F$ u r r y ( $\S 1$ and 2). A general method is developed for calculating the fluctuation in the number of particles after a thickness $x$ of matter $(\S 3)$. It is shown in $\S 4$ that $\overline{N^{2}(x)}$ can always be found when the average energy distribution $F(E, x)$ is known. Using the approximate "cut-off" method the numerical values of $\overline{N^{2}}-(\bar{N})^{2}$ have been computed for the F u r r y model (see table on p. 358). The approach to the normal value of the fluctuation (corresponding to the Pois s o n distribution) is much slower than has been expected.

$\S 1$. Introduction. It is now generally admitted that the interaction laws of electrons and photons with matter are sufficiently well known to form a basis for an explanation of the so-called shower phenomenon. $\mathrm{Carls}$ on and $\mathrm{O}$ p penheimer ${ }^{1}$ ), and $\mathrm{Heit}$ $1 \mathrm{er}$ and $\mathrm{B} \mathrm{h} \mathrm{a} \mathrm{b} \mathrm{a}{ }^{2}$ ) have shown that a combination of the processes of bremsstrahlung and of pair formation gives rise at high energies to a rapid multiplication of particles. In this way they were able to explain the main properties of the "soft" component of cosmic rays. However, there are certain statistical questions which have not been answered completely by these investigations. And since, for the study of the properties of the "hard" component, it is necessary that one be able to subtract with confidence all the effects due to the soft component, we thought it worth while to re-examine the mathematical theory of the shower phenomenon.

It is clear that one has to do with a statistical problem. The proba- 
bilities of the elementary processes being known, one has to find certain distribution functions when a particle traverses a layer of matter causing many of these elementary processes to occur in succession. Till now only the following two questions have been considered.

$A$. An electron of energy $E_{0}$ falls on a layer of matter of thickness $x$; one asks for the average number of particles $F\left(E_{0}, E, x\right) d E$ (electrons or positrons) which will emerge with an energy between $E$ and $E+d E$. This average is meant to be taken over a great number of similar experiments. Since we will neglect as usual the deflections which occur at each elementary process but which are very small at high energies, we will always consider the problem one-dimensional, so that only the dependence on $x$ has to be taken into account. From the function $F$ one finds the average total number of particles:

$$
\bar{N}\left(E_{0}, x\right)=\int_{0}^{E_{0}} d E, F\left(E_{0}, E, x\right)
$$

Experimentally, the function $\bar{N}(x)$ is readily related to the curves obtained by Pfotzer, Bowen, Millikan and Neher for the total intensity of the soft component as a function of the altitude. The theory connects $F\left(E_{0}, E, x\right)$ with the known probabilities for the elementary processes through an integro-differential equation, which is quite similar to the well known $\mathrm{B}$ ol $\mathrm{t} \mathrm{z}$ a $\mathrm{n} \mathrm{n}$ equation in the kinetic theory of gases. In fact, the problem of finding $F$ is analogous to the problem of how the $\mathrm{Maxw}$ a $11-\mathrm{B}$ ol $\mathrm{tz} \mathrm{man} \mathrm{n}$ distribution is reached in time.

$B$. When the incident electron has again the energy $E_{0}$, one must determine the probability $P\left(E_{0}, N, x\right)$, that one will find $N$ particles after the thickness $x$, irrespective of their energy. Experimentally, the function $P(x)$ is readily related to the curves obtained by $\mathrm{R}$ o s$\mathrm{s} i$ and others for the number of coincidences in three or more counters, as a function of the thickness $x$ of matter above the counters. Theoretically, the problem of finding $P\left(E_{0}, N, x\right)$ has some relations to certain little investigated questions in the kinetic theory, which may be formulated as follows. Suppose the average behaviour of a gas which is not in the equilibriun state, is known; what is the fluctuation around this average behaviour? *) From $P$ one finds again

*) Or one may ask: what is the fluctuation around the hyodrodynamical equations of motion. More specifically, this problem implies the question of the calculation of the fluctuation of rates of change, or rates of transport in a gas. 
the average total number $\bar{N}$ according to:

$$
\bar{N}\left(E_{0}, x\right)=\sum_{N} N P\left(E_{0}, N, x\right)
$$

Analogously one can find also $\overline{N^{2}}$, and therefore the fluctuation $\overline{N^{2}}-(\bar{N})^{2}$ of the number of particles.

The calculations of $\mathrm{C}$ a rls on, Op pe n h e i mer, Heit le r and $\mathrm{Bh} \mathrm{a} \mathrm{b} \mathrm{a} \mathrm{are} \mathrm{mainly} \mathrm{concerned} \mathrm{with} \mathrm{the} \mathrm{function} F\left(E_{0}, E, x\right)$. Improvements were made later by $\mathrm{L}$ a $\mathrm{d} \mathrm{a} u$ and $\left.\mathrm{Rumer} \mathrm{r}^{3}\right)$, by $\mathrm{Snyde} \mathrm{r}^{4}$ ) and by $\mathrm{Ser} b$ e ${ }^{5}$ ). The main difficulty has been to take into account the energy losses of the particles due to ionization. Several methods have been proposed, of which the best is certainly the one developed by $\mathrm{Sn}$ y d e r. Problem $B$ was first treated by $\mathrm{F} \mathrm{u} \mathrm{r} \mathrm{r} \mathrm{y}{ }^{6}$ ), who succeeded in giving essentially the solution when one neglects the ionization. He showed that in this case one must expect large fluctuations in the number of particles around $N$; in fact he obtained:

$$
\bar{N}^{2}-(\bar{N})^{2} \cong(\bar{N})^{2}
$$

Further contributions to the solution of Problem $B$ have been made by $A r l e y^{7}$ ) and by $E$ u le ${ }^{8}$ ). They have tried to show that by taking the ionization into account the function $P(N, x)$ will become the $\mathrm{P}$ o i s s o $\mathrm{n}$. distribution for sufficiently large $x$, so that then:

$$
\overline{N^{2}}-(\bar{N})^{2} \cong \bar{N}
$$

We intend partially to review, partially to extend these investigations, using a simplified model, which was first proposed by $\mathrm{F}$ u r r y, and which has all the essential features of the cosmic-ray problem. In $\S 2$ we will describe the F u r y model, and discuss problem $A$ for this case. In $\S \S 3$ and 4 we consider problem $B$ and show that $\bar{N}^{2}$ can always be computed when the solution of problem $A$ is known. This is our main result (Eq. (32)). It enables us to calculate for the F u r r y model the fluctuation $\bar{N}^{2}-(\bar{N})^{2}$ as a function of $x$, when one takes the ionization into account. The result lies between the limits given by (3) and (4), as was to be expected. However, in the interesting region of $x$, (where $\bar{N} \gg 1$ ) the fluctuation turns out to be much larger than the value given by (4), and it approaches this value very slowly when $x$ increases.

§2. Problem $A$ for the Furry model. The model proposed by 
Furry can be described as follows. A particle, colliding with matter, may split into two particles, the sum of whose energies is equal to the energy of the original particle. The probability of the elementary process must be known; we will write

$$
q(E, u) d u d x
$$

for the probability that a particle of energy $E$, while going through the thickness $d x$ of matter, will split into two particles of which one has the energy between $u$ and $u+d u$, while the other has the remaining energy $E-u$. The function $q(E, u)$ must be known; since we do not distinguish between the two particles, $q$ must have the symmetry property:

$$
q(E, u)=q(E, E-u)
$$

We will suppose further that the particles can lose energy by ionization. Let

$$
p(E, u) d u d x
$$

be the probability that in passing through $d x$ the energy of a particlc will go from the value $E$ to the range between $u$ and $u+d u$. The function $p(E, u)$ must also be known. This will be all the processes which can occur *).

The relation between the distribution function $F(E, x)$ and the functions $q(E, u)$ and $p(E, u)$ is given by the continuity equation:

$$
\begin{aligned}
\frac{\partial F}{\partial x}=-F(E, x) \int_{0}^{E} q(E, u) d u+2 \int_{E}^{\infty} q(u, E) F(u, x) d u- \\
-F(E, x) \int_{0}^{E} p(E, u) d u+\int_{E}^{\infty} p(u, E) F(u, x) d u
\end{aligned}
$$

which expresses the fact that in passing through $d x$ the number of particles $F(E, x) d E$ will change because of the losses and gains due to the possible $q$ - and $p$-processes. Eq. (8) has to be solved with the initial condition that for $x=0, F=\delta\left(E-E_{0}\right)$ when $\delta(x)$ is the wellknown singular peak function of $\mathrm{D}$ i r a $\mathrm{c}$.

The problem can be simplified still further by assuming the following properties of the functions $q$ and $p$, which again are quite similar

*) The simplification introduced in the $\mathrm{F}$ u $\mathrm{r} \times \mathrm{y}$ model, compared with the general cosmic ray problem, consists therefore in the omission of the photons and of the bremsstrahlung. 
to the properties of the corresponding functions in the cosmic ray problem:

1. $q(E, u)$ depends only on the fraction of the energy lost; this means that $q(E, u)$ will have the form:

$$
q(E, u)=\frac{1}{E} \chi\left(\frac{u}{E}\right)
$$

where, because of $(6), \chi(\xi)$ must be equal to $\chi(1-\xi)$. A consequence is that the total probability per unit of length for a particle of energy $E$ to split into two, becomes independent of $E$, since:

$$
\int_{0}^{E} q(E, u) d u=\int_{0}^{1} \chi(\xi) d \xi=B
$$

Usually we will take $\chi(\xi)=1$ for simplicity, although this assumption is not necessary.

2. The energy losses through ionization occur every time in very small steps; we will assume therefore that:

$$
p(E, u)=\frac{\beta}{\Delta} \delta(E-u-\Delta)
$$

where $\delta$ is again the $\mathrm{D}$ i r a c function, and $\Delta$ is supposed to be very small compared to $E$ and $u$. The meaning of $\beta$ is the average energy loss of a particle per unit length, since with (11):

$$
\int_{0}^{E}(E-u) p(E, u) d u=\beta
$$

We will suppose that $\beta$ is independent of $E$.

With these assumptions Eq. (8) becomes:

$$
\frac{\partial F}{\partial x}=-B F(E, x)+2 \int_{E}^{\infty} \frac{d u}{u} \chi\left(\frac{E}{u}\right) F(u, x)+\beta \frac{\partial F}{\partial E}
$$

when we go to the limit $\Delta \rightarrow 0$. In this form the problem can be solved when one may neglect the ionization, i.e. for $\beta=0$. Following $\mathrm{L}$ a $\mathrm{nd} \mathrm{a} u$ and $\mathrm{Rumer}$ we introduce the moments

$$
f(s, x)=\int_{0}^{\infty} E^{s} F(E, x) d E
$$

From (12) one then easily obtains (always with $\beta=0$ !) the following 
differential equation for $t$ :

$$
\frac{\partial f(s, x)}{\partial x}=[2 A(s)-B] f(s, x)
$$

when:

$$
A(s)=\int_{0}^{1} \xi^{s} \chi(\xi) d \xi
$$

Since for $x=0, F=\delta\left(E-E_{0}\right)$, so that $f=E_{0}^{s}$ one gets for the moments:

Especially:

$$
f(s, x)=E_{0}^{s} e^{[2 A(s)-B] x} .
$$

so that:

$$
A(0)=B, A(1)=\frac{1}{2} B
$$

$$
\begin{gathered}
f(0, x)=\bar{N}(x)=e^{B x} \\
f(1, x)=E_{0}
\end{gathered}
$$

The total energy of the particles remains therefore $E_{0}$, while the number of particles increases exponentially. From (16) one can obtain the energy distribution $F(E, x)$ by using the $\mathrm{M}$ ell in transformation:

$$
F(E, x)=\frac{1}{2 \pi i} \int_{-i \infty}^{+\infty} d s f(s, x) E^{-s-1}
$$

where the path of the integration has to be taken on the right of the singularities of the integrand. For instance, with $\chi(\xi)=1, B=1$, $A(s)=1 /(s+1)$, one gets:

$$
\begin{aligned}
F\left(E_{0}, E, x\right)=\frac{1}{2 \pi i} \frac{e^{-x}}{E_{0}} \int_{-i \infty}^{+\infty} d s\left(\frac{E_{0}}{E}\right)^{s+1} e^{2 x / s+1} & = \\
& =e^{-x}\left[\delta\left(E-E_{0}\right)+\frac{4 x}{E_{0} y} I_{1}(y)\right]
\end{aligned}
$$

where $y^{2}=8 x \log \left(E_{0} / E\right)$ and $I_{1}(y)$ is the B e s s e 1 function of first order and imaginary argument. The energy distribution consists therefore of a diminishing peak at $E_{0}$ and a part which increases monotonically from a finite value at $E=E_{0}$ to infinity at $E=0$.

When one wants to take the ionization into account the problem becomes much more difficult. We hope to return to this question in a subsequent article, where we will also discuss the method proposed 
by $\mathrm{S} n$ y d e r. Here we will content ourselves with a rough approximation method first used by $\mathrm{H}$ e i t $\mathrm{l}$ e $\mathrm{r}$ and $\mathrm{B} \mathrm{h}$ a b a. To express the influence of the ionization on the average number of particles $\bar{N}(x)$, these authors integrate the energy distribution without ionization (given by (17) resp. (17a)) from a fixed lower limit $\varepsilon$ to $E_{0}$. As a consequence, instead of increasing with $x$, the $\bar{N}(x)$ will now go through a maximum and drop to zero for large $x$. It is clear that for every $x$ one can find a value of $\varepsilon$ which will give the right answer, and that for larger $\beta$ one has to take also a larger $\varepsilon$. Since with this "cut-off" method one overestimates the number of fast particles, one should take a bigger cut-off energy $\varepsilon$ for larger values of $x$, so that $\varepsilon$ will be an increasing function both of $\beta$ and of $x$. We will neglect this variation however, and consider $\varepsilon$ as a constant of which the value can still be adjusted $*$ ). With $\chi(\xi)=1$ one then easily finds from $(17 a)$ :

$$
\bar{N}(z, x)=\frac{e^{-x}}{2 \pi i} \int_{\delta \rightarrow i \infty}^{\delta+i \infty} \frac{d s}{s} e^{s z+(2 x / s+1)}
$$

when $z=\log \left(E_{0} / \varepsilon\right)$ and the path of integration is taken to the right of the origin. By bending the path towards the left this can be transformed to:

$$
\bar{N}(z, x)=e^{-x}+e^{-x} \int_{0}^{y} d \eta e^{-\left(\eta^{2} / 8 x\right)} I_{1}(\eta)
$$

when $y^{2}=8 x z$. Figures 1 and 2 show $\bar{N}(x)$ for $z=4.75$ and $z=7$, computed from (18a) by numerical integration. One can evaluate (18) also by the method of steepest descent, shifting the path of integration to the saddle point $s_{0}$ of the exponential factor, which is given by:

$$
s_{0}+1=\sqrt{\frac{2 x}{z}}
$$

and one then obtains in the usual way:

$$
\bar{N}(z, x) \cong \frac{e^{-x-z+y}}{\sqrt{2 \pi y}} \frac{1}{1-\sqrt{\frac{z}{2 x}}}=N_{0}(z, x)
$$

*) $\varepsilon$ will be roughly proportional to $\beta$; in the cosmic ray problem it turns out that one gets the best results by taking $\varepsilon \approx 0.4 \beta$. 
This will be a good approximation only for sufficiently large $x$ and $z^{*}$ ). By comparing (18b) with (18a) (see Figs. 1 and 2) one sees that the method of steepest descent gives rather accurate results even for

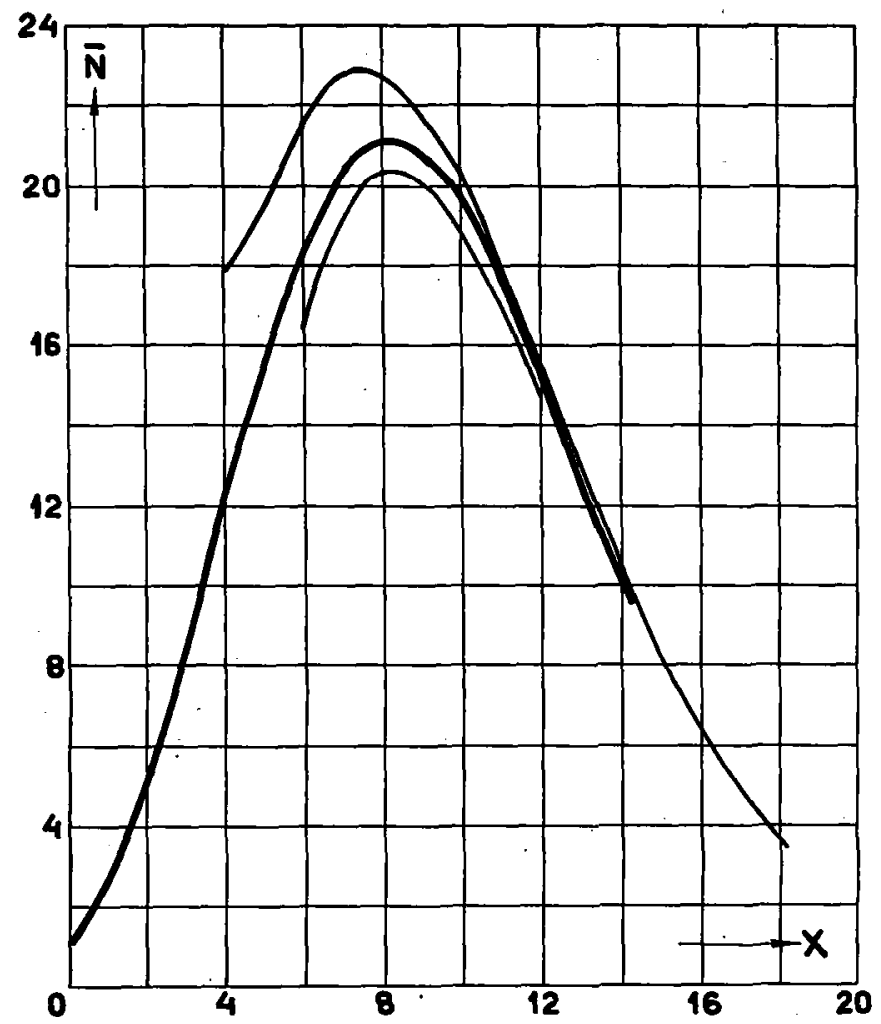

Fig. 1. Average number of particles as function of the thickness according to the cut-off method; $z=4.75$. Heavy curve calculated from Eq. (18a); upper curve by using the method of steepcst descent (Eq. (18b)); lower curve by using the next approximation to this method (Eq. (18i)).

values of $x$ near the maximum of $\bar{N}(x)$. For smaller values of $x$ however, the approximation (18b) breaks down. From (18b) one may

*) The pararneters involved are more precisely $y$ and $s / 2 x$. By improving the saddle point method one can derive:

$$
\bar{N}(z, x) \backsim N_{0}(z, x)\left[1-\frac{3}{8 y}-\frac{1}{y\left(1-\sqrt{\frac{\dot{z}}{2 x}}\right)^{2}}+\frac{3}{2 y} \frac{1}{1-\sqrt{\frac{z}{2 x}}}\right] .
$$

This shows that near the maximum the error in (18b) will be about $10 \%$. 
derive therefore the position and the value of the maximum of $\bar{N}(x)$ and one finds:

$$
\begin{gathered}
x_{m} \cong 2 z-\frac{3}{2} \\
\bar{N}\left(x_{m}\right) \cong \frac{e^{z}}{\sqrt{2 \pi z}} .
\end{gathered}
$$

The position of the maximum increases therefore logarithmically with the incoming energy $E_{0}$, while the value of the maximum is roughly proportional to $E_{0}$.

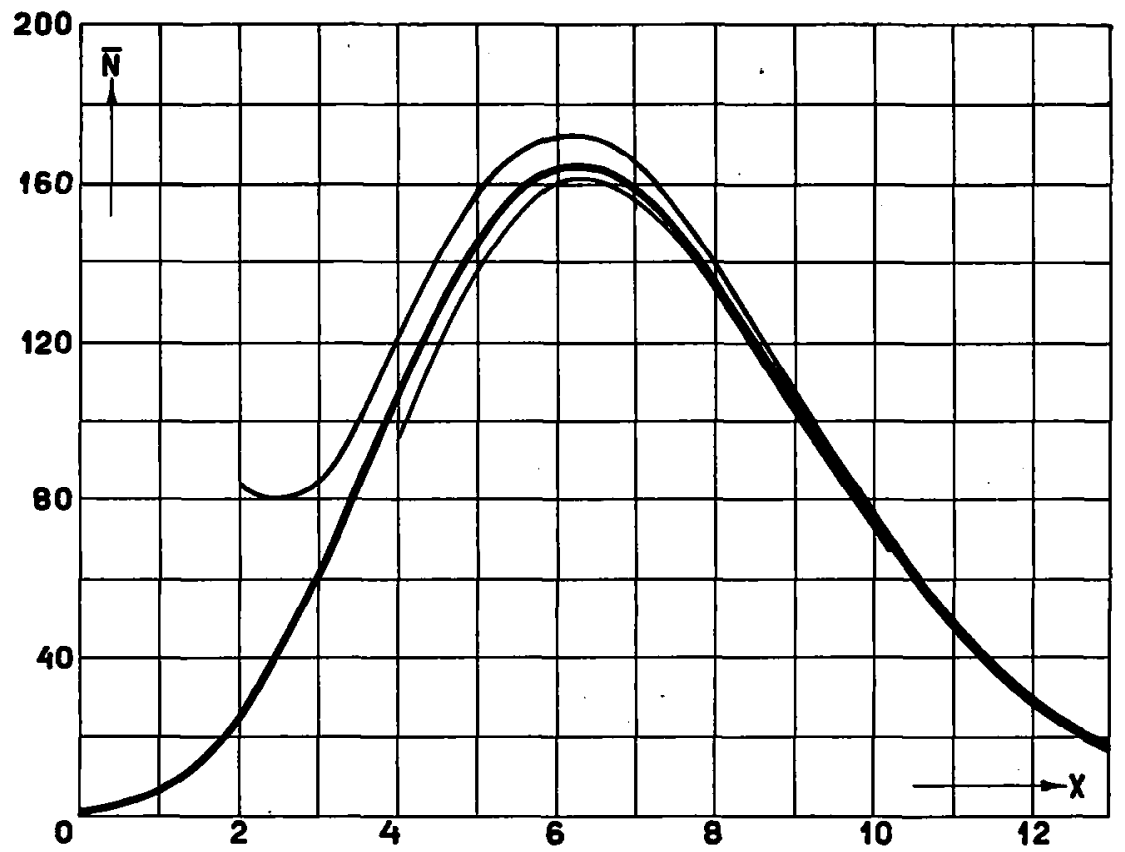

Fig. 2. The same as Fig. 1 except that now $z=7$.

§ 3. The "master" equation. Before turning to problem B, we will consider first the most general question, which can be stated and which contains the problems A and B as special cases. Suppose that we have again an incident electron of energy $E_{0}$; we may ask then for the probability of a certain energy distribution of the particles, which emerge after the thickness $x$. To formulate this problem more precisely, let us assume for simplicity *) that the energy of the part-

*) Otherwise we should have to work with functional equations! 
icles can have only the discrete values $\varepsilon_{1}, \varepsilon_{2}, \varepsilon_{3}, \ldots$ An energy distribution is then specified by giving the numbers of particles $n_{1}, n_{2}, n_{3}$, $\ldots$ which have these energy values. The required probability of an energy distribution will be a function of the numbers $n_{i}$ and of $x$, which we will denote by $W\left(n_{1}, n_{2}, \ldots ; x\right)$. From this function $W$ one can find all other distribution functions. For instance, the average number of particles of energy $\varepsilon_{i}$ is given by:

$$
\bar{n}_{i}(x)=S n_{i} W\left(n_{1}, n_{2} \ldots ; x\right)
$$

where the round summation sign will always mean a sum over all values of $n_{1}, n_{2}, \ldots$. For a continuous energy distribution $\bar{n}_{i}(x)$ clearly goes over into the function $F(E, x)$ of problem A. Analogously:

$$
P(N, x)=S^{\prime} W\left(n_{1}, n_{2} \ldots ; x\right)
$$

where the accent means that one has to sum over the $n_{i}$ with the condition:

$$
\sum_{i} n_{i}=N
$$

When the probabilities of the elementary processes are known, one can write down a continuity equation for $W$, from which all other equations can be derived and which we will call therefore the ",master" equation. For the F u r r y model one finds:

$$
\begin{aligned}
\frac{\partial W}{\partial x}- & -W \sum_{k} n_{k} B_{k}+\sum_{i j} q_{i j}\left(n_{i}+1\right) W\left(n_{j}-1, n_{i-j}-1, n_{i}+1 ; x\right) \\
& -W \sum_{k} n_{k} R_{k}+\sum_{i j} p_{i j}\left(n_{i}+1\right) W\left(n_{j}-1, n_{i}+1 ; x\right)
\end{aligned}
$$

Here, analogous to (5) and (7), $q_{i j} d x$ and $p_{i j} d x$ represent the probabilities for a splitting and for an energy loss when a particle goes through a layer $d x$ of matter. Analogous to (6):

$$
q_{i j}=q_{i-j}
$$

Finally:

$$
B_{i}=\sum_{i} q_{i j} \quad R_{i}=\sum_{i} p_{i j} .
$$

As an example we will derive $\mathrm{F}$ u r r y's result for $P(N, x)$. For this we have to assume:

1. Only $q$-processes occur.

2. $B_{k}$ is independent of $k$; as we saw, this is a consequence of assumption (9) for the splitting probability $q_{i j}$. With the definition 
(21) for $P(N, x)$ one finds then easily from the first line of $(22)$ :

$$
\frac{\partial P(N, x)}{\partial x}=-B N P(N, x)+B(N-1) P(N-1, x)
$$

For $x=0, P(N, x)=0$ except $P(1, x)$ which is equal one. With this initial condition the solution of (23) becomes:

$$
P(N, x)=e^{-B x}\left(1-e^{-B x}\right)^{N-1}
$$

$P(N, x)$ is therefore a monotonic decreasing function of $N$. The most probable number of particles is always equal to one. For the average number of particles one finds:

$$
\bar{N}(x)=\sum_{N} N P(N, x)=e^{B x}
$$

in agreement with (16). For the fluctuation one obtains:

$$
\overline{N^{2}}-(\bar{N})^{2}=(\bar{N})^{2}-\bar{N}
$$

Unfortunately it is not possible to generalize Eq. (23) for the F u r$\mathrm{r} y$ model with inonization, even if one makes for the $p$-processes the assumption (11).

$\S 4$. The general expression for $\overline{N^{2}}$. Restricting oneself to the successive calculation of the average values $\bar{N}, \bar{N}^{2}$ etc., one can proceed as follows. From the master equation (22) one derives easily:

$$
\begin{gathered}
\frac{d}{d x} S W=0 \\
\frac{d \bar{n}_{i}}{d x}=-B_{i} \bar{n}_{i}+2 \sum_{j} q_{j i} \bar{n}_{i}-R_{i} \bar{n}_{i}+\sum_{i} p_{i i} \bar{n}_{i}
\end{gathered}
$$

The first equation says that the total probability does not vary with $x$ and can therefore be normalized to one. The second equation shows how the average number of particles of energy $\varepsilon_{i}$ changes with $x$. For a continuous energy distribution $(25 b)$ is clearly identical with (8). In this way we can go on. The next step is the continuity equation for the quadratic averages:

$$
\overline{n_{i} n_{j}}=S n_{i} n_{j} W .
$$

For a continuous energy distribution this will become a function of two energy variables and of $x$, which we will denote by $G\left(E_{1}, E_{2}, x\right)$. Because of its length we will not write down the equation which one can derive for $G$ from the master equation (22). It is a linear, inhomo- 
geneous, integral equation, in which the inhomogeneous part has some terms containing the singular function $\delta\left(E_{1}-E_{2}\right)$. One has to solve this equation with the initial condition that for $x=0$ :

$$
G\left(E_{1}, E_{2}, 0\right)=\delta\left(E_{1}-E_{0}\right) \delta\left(E_{2}-E_{0}\right)
$$

which represents the fact that for $x=0$ one knows that there is one particle of energy $E_{0}$. The meaning of the equation for $G$ becomes quite clear, when one splits off from $G$ a singular part containing $\delta\left(E_{1}--E_{2}\right)$. One can show then very easily that the equation for $G$ is fulfilled by the "Ansatz":

$$
G\left(E_{1}, E_{2}, x\right)=\delta\left(E_{1}-E_{2}\right) F\left(E_{1}, x\right)+K\left(E_{1}, E_{2}, x\right)
$$

Here $F$ is the same function as occurs in $\S 2$; it fulfills Eq. (8) and for $x=0$ it has to become $\delta\left(E_{1}-E_{0}\right)$. The function $K$ is regular for $E_{1}=E_{2} ;$ for $x=0$ it has to become zero, so that $G$ clearly fulfills the initial condition (26). The equation for $K$ can be written in the form:

$$
\frac{\partial K}{\partial x}=\left(L_{E_{1}}+L_{E_{2}}\right) K\left(E_{1}, E_{2}, x\right)+2 F\left(E_{1}+E_{2}, x\right) q\left(E_{1}+E_{2}, E_{1}\right)
$$

To abbreviate, we have denoted here by $L_{E}$ the linear operator which acts on the variable $E$ in $F(E, x)$ on the right hand side of Eq. (8). With the same notation the equation for $F$ would be written as:

$$
\frac{\partial F}{\partial x}=L_{E} F(E, x)
$$

The equation for $K$ is therefore again a linear inhomogeneous integral equation. Without the inhomogeneous part the equation would be separable, and a solution would be $F\left(E_{1}, x\right) . F\left(E_{2}, x\right)$. Substituting in (27), we see that there would then be no correlation between the numbers of particles in different energy intervals $*$ ). This correlation is therefore due to the inhomogeneous part of Eq. (28), and we may say that the numbers of particles in two different energy intervals $\left(E_{1}, E_{1}+d E_{1}\right)$ and $\left(E_{2}, E_{2}+d E_{2}\right)$ are statistically coupled with each other because of the fact that there are particles of energy $E_{1}+E_{2}$, which can split and produce particles of energies $E_{1}$ and $E_{2}$. The general solution of (28) with the initial condition $K\left(E_{1}, E_{2}, 0\right)=$

*) For the discrete energy distribution we would get:

$$
\overline{n_{i} n_{j}}=\delta_{i j} \bar{n}_{i}+\bar{n}_{i} \cdot \bar{n}_{j}
$$

which is the well-known result for the case that the states $i$ and $j$ are statistically independent. 
$=: 0$ can be obtained by observing that $F\left(E_{0}, E_{1}, x\right) . F\left(E_{0}, E_{2}, x\right)$ is not only a solution of the homogeneous equation but that it is also the fundamental solution, since for $x=0$ it becomes $\delta\left(E_{0}-E_{1}\right)$. $\delta\left(E_{0}-E_{2}\right)$. One can therefore always find the solution of the inhomogeneous equation. In our case it is given by:

$$
\begin{aligned}
K\left(E_{1}, E_{2}, x\right)=2 \int_{0}^{\infty} \int_{0}^{\infty} d \xi d \eta \int_{0}^{x} d \tau F\left(E_{0}, \xi+\eta, \tau\right) q(\xi+\eta, \xi) . \\
. F\left(\xi, E_{1}, x-\tau\right) F\left(\eta, E_{2}, x-\tau\right)
\end{aligned}
$$

as can easily be verified. From the function $G$ one finds the average square of the total number of particles according to:

$$
\overline{N^{2}}\left(E_{0}, x\right)=\iint_{0}^{E_{0}} d E_{1} d E_{2} G\left(E_{1}, E_{2}^{\cdot}, x\right)
$$

Using (27), (30) and the definition (1) for $\bar{N}\left(E_{0}, x\right)$, this becomes *): $\overline{N^{2}}\left(E_{0}, x\right)=\bar{N}\left(E_{0}, x\right)+2 \int_{0}^{\infty} \int d \xi d \eta \int_{0}^{x} d \tau F\left(E_{0}, \xi+\eta, \tau\right) q(\xi+\eta, \xi)$.

$$
. \bar{N}(\xi, x-\tau) \bar{N}(\eta, x-\tau)
$$

$\overline{N^{2}}$ can therefore be evaluated, when the function $F$ is known. It seems likely that this will hold also for the averages of all higher powers of $N$.

$\S 5$. Calculation of $\overline{N^{2}}$ with the cut-off method. In order to get definite numerical results for the fluctuation, we will again make the assumptions (9) (with $\chi=1$ ) and (11) for the probabilities of the $q$ and $p$-process. According to the idea of the cut-off method we must then first find $G\left(E_{1}, E_{2}, x\right)$ for $\beta=0$. This can be done by introducing the expression (17a) for $F$ in (30). The integrations over $\xi$ and $\eta$ can then be carried out and one obtains:

$$
\begin{array}{r}
K\left(E_{1}, E_{2}, x\right)=\frac{2 e^{-2 x}}{(2 \pi i)^{2}} \iint d s d t \frac{E_{0}^{s+t}}{E_{1}^{s+1} E_{2}^{t+1}} \frac{\Gamma(s+1) \Gamma(t+1)}{\Gamma(s+t+2)} . \\
. e^{2 x(1 / s+1)+(1 / t+1))} \int_{0}^{x} d \tau e^{2 B(s, t) \tau}
\end{array}
$$

where:

$$
B(s, t)=\frac{1}{s+t+1}+\frac{1}{2}-\frac{1}{s+1}-\frac{1}{t+1}
$$

and the paths of the integration in the $s$-and $t$-plane have to be taken

*) Remember for instance that $F\left(\xi, E_{1}, x-\tau\right)$ is certainly zero for $E_{1}>\xi$. 
parallel to the imaginary axis and on the right of the-singularities of the integrand.

Another way of deriving (33) is by generalizing the method of moments used in $\S 2$ for the $F$-equation. Defining:

$$
k(s, t, x)=\int_{0}^{\infty} \cdot \int_{1}^{s} E_{2}^{t} K\left(E_{1}, E_{2}, x\right)
$$

one finds from (28) the inhomogeneous linear differential equation:

$$
\left.\frac{\partial k}{\partial x}=2[A(s)+A(t)-B] k+2 C(s, t) f(s+t), x\right)
$$

where $B, A(s)$ and $f(s, x)$ are again given by (10), (15) and (13), while.

$$
C(s, t)=C(t, \mathrm{~s})=\int_{0}^{1} d \xi \xi^{s} \cdot(1-\xi)^{t} \chi(\xi)
$$

One has to solve Eq. (36) with the initial condition $k=0$ for $x=0$ From $k(s, t, x)$ one finds $K\left(E_{1}, E_{2}, x\right)$ by using a double $\mathrm{M}$ e $11 \mathrm{in}$ transformation. With $\chi(\xi)=1$ one obtains then again Eq. (33).

According to the cut-off method we have now to integrate (33) over $E_{1}$ and $E_{2}$ between the limits $\varepsilon$ and $E_{0}$. Writing again $z=\log$ $\left(E_{0} / \varepsilon\right)$ one finds then:

$$
\begin{gathered}
\overline{N^{2}}(z, x)=\bar{N}(z, x)+2 e^{-2 x} \int_{0}^{x} d \tau e^{\tau} I(z, x, \tau) \\
(z, x, \tau)=\frac{1}{(2 \pi i)^{2}} \iint d s d t \frac{\Gamma(s) \Gamma(t)}{\Gamma(s+t+2)} e^{(s+t) z+(2 \tau / s+t+1)+2(x-\tau)((1 / s+1)+(1 / t+1))}
\end{gathered}
$$

where the paths of integration over $s$ and $t$ have now to be taken to the right of the origin.

We have evaluated the double complex integral with the saddle point method, considering only the exponential factor as rapidly varying. From the experience gained in $\S 2$ one can expect only good results for values of $x$ greater than $x_{m}$ (given by (19)). We have therefore taken $x=2 z, 3 z$ and $4 z$. Even then it turns out that the saddle point method breaks down for values of $\tau$ near the upper limit $x$, so that in this region one has to use another method. The integral over $\tau$ has to be carried out numerically. The details of the calculation for the case $x=2 z$ are given in the appendix. The results are recorded in the following table: 


\begin{tabular}{|c|c|c|c|c|c|c|c|c|}
\hline \multicolumn{8}{|c|}{$z=4.75$} & \multicolumn{6}{|c|}{$z=7$} \\
\hline$x$ & $\bar{N}$ & $f$ & $\sigma$ & $x$ & $\bar{N}$ & $f$ & $\sigma$ \\
\hline $2 z$ & 20.4 & 1.42 & 9.6 & $2 z$ & 159 & 1.26 & 42 \\
$3 z$ & 9.7 & 1.57 & 6.5 & $3 z$ & 60 & 1.47 & 29 \\
$4 z$ & 2.5 & 1.35 & 4.4 & $4 z$ & 9 & 1.72 & 16.5 \\
\hline
\end{tabular}

Here we have written:

$$
\overline{N^{2}}=\bar{N}+f(z, x)(\bar{N})^{2}
$$

while $\sigma$ is defined by:

$$
\sigma=\frac{\overline{N^{2}}-(\bar{N})^{2}}{\bar{N}}
$$

From the general considerations of $\S 4$ one must expect that for very small $x$ the fluctuation is given by $\mathrm{F} \mathrm{u} \mathrm{r}$ r y's formula (3) (resp. (3a)), so that then $\sigma \cong \bar{N}-1$, while for very large $x$ the normal fluctuation law (4) should hold, so that $\sigma \rightarrow 1$. The results found lie between these two limits, except for $x=4 z$. Unfortunately the results for $x=4 z$ are the least accurate (esp. for $z=4.75$ ), since the region $\tau \cong x$ becomes more and more important in the integral (38). However, we think that one cannot escape the conclusion that the $\sigma(x)$ curve approaches the asymptotic value $\sigma=1$ very slowly, slower even than the $\bar{N}(x)$ curve approaches the value zero. We have therefore to expect large fluctuations even for the tail of the multiplication curve $\bar{N}(x)$.

§6. Appendix. Take in Eq. (38) $x=2 z$, and put $\tau=\alpha x$. Because of the symmetry of the integrand in $s$ and $t$ we will get the same saddle points $s_{0}=t_{0}$ in the $s$ - and in the $t$-plane. Write $p(\alpha)=s_{0}+$ $+1=t_{0}+1$. Since we will consider only the exponential factor as rapidly varying, we obtain for $p(\alpha)$ the fourth order equation:

$$
1-\frac{4 \alpha}{(2 p-1)^{2}}-\frac{4(1-\alpha)}{p^{2}}=0 .
$$

This has to be solved for all values of $\alpha$ between zero and one. For $\alpha=0, p=2$, and for small values of $\alpha$ one easily finds the series development:

$$
p(\alpha)=2-\frac{5}{9} \alpha+0.0052 \alpha^{2}+0.035 \alpha^{3} \ldots
$$


For $\alpha=1, p=1.5$, and for small $\varepsilon=1-\alpha$ one finds again:

$$
p(\alpha)=1.5+\frac{7}{18} \varepsilon+0.150 \varepsilon^{2}-0.0133 \varepsilon^{3} \ldots
$$

With these series one can compute $p(\alpha)$ to about three decimal places.

By applying the ordinary saddle point method and by using for $\bar{N}$ the expression $(18 b)$ of $\S 2$, one can then write for the factor $f(z, x)$ occurring in the formula (39):

$$
f(z, x=2 z)=\frac{z}{2} \int_{0}^{1} \frac{d \alpha}{\sqrt{1-\alpha}} \frac{\Gamma^{2}(p-1)}{\Gamma(2 p)} \frac{1}{\sqrt{g(\alpha)}} e^{2 z \phi(a)}
$$

where:

$$
\begin{gathered}
\varphi(\alpha)==p+\alpha-4+\frac{2 \alpha}{2 p-1}+\frac{4(1-\alpha)}{p} \\
g(\alpha)=\frac{1-\alpha}{p^{6}}+\frac{2 \alpha}{p^{3}(2 p-1)^{3}} .
\end{gathered}
$$

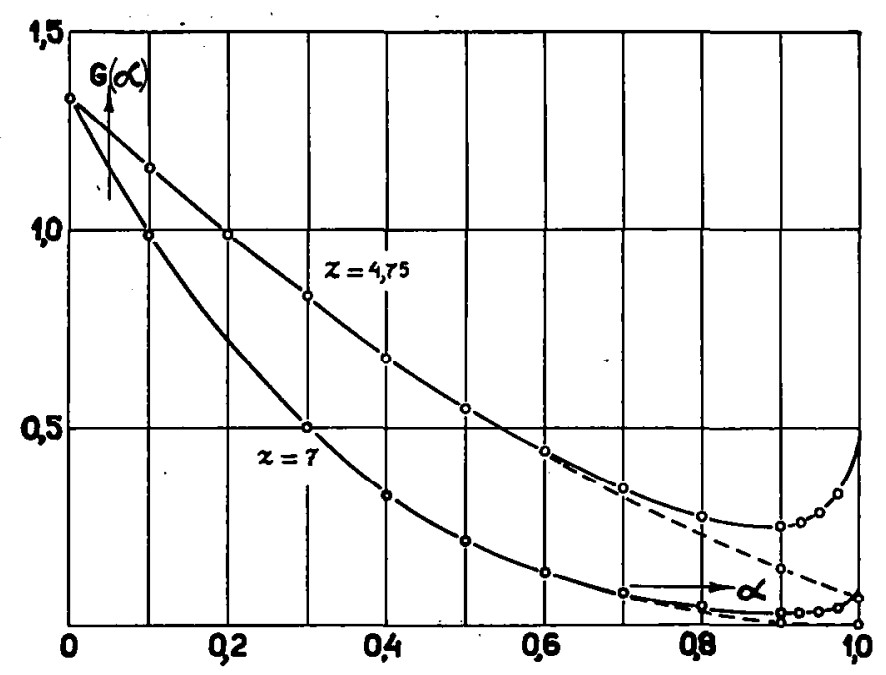

Fig. 3. The integrand of Eq. (43) as a function of $\alpha$.

Using for $p(\alpha)$ the value computed from (41) and (42), we have plotted in Fig. 3 the integrand of (43) (denoted by $G(\alpha, z)$ ) as a function of $\alpha$ for $z=4.75$ and $z=7$. Since for $\alpha=1, G$ becomes infinite as $1 /(1-\alpha)^{\ddagger}$ one has to expect that near $\alpha=1$ the saddle point 
method breaks down. To calculate $G$ in this region, we start again from (38). For $x=2 z$ and $\alpha=1$, the complex integral becomes:

$$
I(z, 2 z, 2 z)=\frac{1}{(2 \pi i)^{2}} \iint d s d t e^{(s+t) s+(4 z / s+t+1)} \frac{\Gamma(s) \Gamma(t)}{\Gamma(s+t+2)} .
$$

Put $s=\sigma+\tau, t=\sigma-\tau$, then the integral over $\tau$ becomes:

$$
\int_{-i \infty}^{+\infty} d \tau \Gamma(\sigma+\tau) \Gamma(\sigma-\tau)=\frac{\Gamma(2 \sigma)}{2^{2 \sigma}} .
$$

The integral over $\sigma$ can be done by the saddle point method, and one obtains:

$$
G(1, z)=\frac{8 z \sqrt{2 \pi}}{\sqrt[4]{z(z-\log 2)}} e^{-5 x+4 \sqrt{z(z-\log 2)}} \frac{1}{2 \sqrt{\frac{z}{z-\log 2}}-1} .
$$

In this fashion one can determine also the value of $G$ near $\alpha=1$. Putting $\varepsilon=1-\alpha$, one finds approximately:

$$
G(\alpha, z)=G(1, z)\left[1+0.912 \varepsilon z+0.589 \varepsilon^{2} z^{2} \ldots\right] .
$$

With the values thus obtained, one can extrapolate with confidence the $G(\alpha)$ curve towards $\alpha=1$. The value of the integral must then be determined graphically.

Recejved Feluruary 23rd, 1940.

\section{REFERENCES}

1) J.F. Carls on and J. R. O p pen hei mer, Phys. Rev, 51, 220 (1937).

2) H. J. B b a b b and $W$. H e it le r, Proc. roy. Soc. 159, 432 (1937).

3) L. L a n d a u and $G$. $R$ a $m$ e r, Proc. roy. Soc. 166, 213 (1938).

4) H. S n y der, Phys. Rev. 53, 960 (1938).

5) R. Serber, Phys. Rev. 54, 317 (1938).

6) W. H. F uriv, Phys. Rev. 52, 569 (1937).

7) N. A r le y, Proc. roy. Soc. 168, 519 (1938).

8) HT. E ule r, 7.. Pliys. 110, 450 (1938). 